\title{
Spoof-Fluid-Spoof Acoustic Waveguide and its Applications for Sound Manipulation
}

\author{
Norbert Cselyuszka $\odot,{ }^{1,2, *}$ Andrea Alù, ${ }^{3,4,5,6}$ and Nikolina Janković ${ }^{1}$ \\ ${ }^{1}$ BioSense Institute, University of Novi Sad, Novi Sad, Serbia \\ ${ }^{2}$ Silicon Austria Labs, Sensor System, Microsystem Technologies, Villach, Austria \\ ${ }^{3}$ Department of Electrical and Computer Engineering, The University of Texas at Austin, Austin, Texas 78712, \\ USA \\ ${ }^{4}$ Photonics Initiative, Advanced Science Research Center, City University of New York, New York, \\ New York 10031, USA \\ ${ }^{5}$ Physics Program, Graduate Center, City University of New York, New York, New York 10026, USA \\ ${ }^{6}$ Department of Electrical Engineering, City College of New York, New York, New York 10031, USA
}

(Received 12 July 2019; revised manuscript received 14 October 2019; published 6 November 2019)

\begin{abstract}
We explore the implementation of a spoof-fluid-spoof (SFS) acoustic waveguide, which is the acoustic analogue of a metal-insulator-metal waveguide in plasmonics. The proposed structure consists of two corrugated rigid surfaces separated by a fluid layer. The properties of the guided acoustic modes between corrugated rigid parallel plates are analytically and numerically studied by deriving their dispersion relation and through a careful analysis of symmetric and antisymmetric modes supported by this structure. Similar to plasmonic waveguides, it is shown that the dispersion relation of the proposed SFS acoustic waveguide, and the supported group and phase velocities, can be largely controlled by changes in the geometrical parameters of the waveguide, opening the possibility for application of this technology for acoustic delay lines, modulators, and sensors. The analytical and numerical results are validated through experiments in a controlled environment.
\end{abstract}

DOI: 10.1103/PhysRevApplied.12.054014

\section{INTRODUCTION}

Surface plasmon polaritons (SPP) are electromagnetic waves supported at the interface of a dielectric material and noble metals due to the coupling of light with free electron oscillations [1]. Because of their specific nature, SPPs can break the diffraction limit and localize light into deeply subwavelength dimensions. As such, SPPs have been applied in various fields, including optical communications, photonics, and sensing [2-5]. Since SPPs naturally occur only at optical frequencies, different concepts have been proposed to engineer SPP phenomena in other frequency ranges, including microwaves and terahertz. One of the concepts that effectively mimics SPPs are spoof surface plasmon polaritons [6,7], which are supported by specifically tailored structures, such as corrugated metallic surfaces whose geometrical properties can be used to

\footnotetext{
*norbert.cselyuszka@silicon-austria.com
}

Published by the American Physical Society under the terms of the Creative Commons Attribution 4.0 International license. Further distribution of this work must maintain attribution to the author(s) and the published article's title, journal citation, and DOI. tailor the guided waves and mimic SPPs in terms of field localization and slow-wave properties.

Among the most common plasmonic waveguide structures, metal-insulator-metal (MIM) waveguides, composed of two metallic claddings embedding a dielectric core, have received special attention for guiding SPPs [8-10]. The spoof plasmon analogue of MIM is the doubly-corrugated waveguide, which comprises a spoofinsulator-spoof (SIS) waveguide [11,12]. Following the concept of electromagnetic spoof SPPs, the analogue acoustic phenomenon was proposed and investigated in several papers [13-29]. In contrast to conventional acoustic surface waves in which the waves propagate on the surface of solids and the properties are closely dependent on the material parameters [30], spoof acoustic surface waves propagate along the interface between the background medium and a corrugated rigid plate due to coupling between the oscillation inside the individual holes and the mutual near-field coupling between the adjacent holes. It has been shown that the geometrical parameters of the corrugations provide a large degree of freedom to control the dispersion properties, which has led to a number of applications, such as collimation of sound [15-19], acoustic rainbow trapping [20,21], acoustic sensing enhancement [22], acoustic wave 
focusing [25], and temperature-controlled acoustic surface waves [29].

Although acoustic waveguides play an important role in various technological platforms, spoof acoustic surface waves have not been investigated so far. In this paper, the acoustic counterpart of the plasmonic MIM structure based on the acoustic spoof plasmon concept is presented. Two corrugated rigid surfaces separated by a gap filled with a fluid medium form the spoof-fluid-spoof (SFS) acoustic waveguide. The proposed structure is analyzed in detail using effective medium theory and the dispersion relation for the waveguide is derived. We also show how acoustic wave propagation can be controlled in the proposed waveguide by changing the gap height, which opens the possibility for applications in the domain of sensing and acoustic wave modulation. To validate our analytical and numerical results, the proposed structure is fabricated using three-dimensional- (3D) printing technology and measured in a controlled environment, showing excellent agreement with our theoretical and numerical predictions.

\section{DISPERSION EQUATION OF A SFS ACOUSTIC WAVEGUIDE}

The proposed geometry is based on a doubly corrugated waveguide, consisting of two perfectly rigid slabs with periodic grooves separated by a gap Fig. 1(a). The parameters $d, a$, and $h$ stand for the period, width, and depth of the grooves, respectively, while $g$ is the height of the fluid gap between the rigid corrugated surfaces. For the sake of simplicity and without losing generality, we consider a lossless two-dimensional (2D) case, where the structure extends infinitely along the $z$ direction and the acoustic wave propagates in the $x-y$ plane.

The dispersion relation of the SFS waveguide is derived using an effective medium approximation, which is valid when the period of the grooves $d$ is much smaller than the guided wavelength, $\lambda \gg d$. In this regime, the periodic corrugated surface can be considered an equivalent anisotropic homogeneous medium characterized by an effective density tensor. Consequently, the SFS structure can be replaced with an equivalent three-layer structure with effective material properties, bounded by perfectly rigid walls from the top and bottom sides at the planes $y=0$ and $y=b$. The equivalent structure with corresponding dimensions is shown in Fig. 1(b).

In the $2 \mathrm{D}$ scenario, assuming symmetry and infinite extent in the $z$ direction, the relevant components of mass density and bulk modulus tensors for the corrugated surface are labeled $\rho_{y}^{j}, \rho_{x}^{j}, \rho_{y}^{j}$, and $\kappa$, respectively, where $j$ takes the values I, II, and III, denoting the top, middle, and bottom layers, respectively. For the air-filled corrugated structure, the effective material parameters can be defined as $\rho_{y}^{\mathrm{I}}=\rho_{y=}^{\mathrm{III}}(d / a) \rho_{\text {air }}, \rho_{x}^{\mathrm{I}}=\rho_{x}^{\mathrm{III}} \rightarrow \infty$, while $\kappa_{\text {eff }}=(d / a) \kappa_{\text {air }}$ [31]. In the middle layer, the effective parameters are equal to the material parameters of the fluid, that is, in this case to air: $\rho^{\mathrm{II}}=\rho_{\text {air }}=1.21 \mathrm{~kg} / \mathrm{m}^{3}$ and $\kappa_{\text {air }}=1.41 \cdot 10^{5} \mathrm{~Pa}$. The dispersion equations for the SFS waveguide can be written as

$$
\begin{aligned}
& \frac{a}{d} k_{0} \tan \left(h k_{0}\right)=\sqrt{\beta^{2}-k_{0}^{2}} \tanh \left(\frac{g}{2} \sqrt{\beta^{2}-k_{0}^{2}}\right), \\
& \frac{a}{d} k_{0} \tan \left(h k_{0}\right)=\sqrt{\beta^{2}-k_{0}^{2}} \operatorname{coth}\left(\frac{g}{2} \sqrt{\beta^{2}-k_{0}^{2}}\right),
\end{aligned}
$$

where $k_{0}=\omega \sqrt{\rho_{\text {air }} / \kappa_{\text {air }}}$ is the wave number in the fluid and $\beta$ is the propagation constant in the SFS waveguide. Derivation of Eqs. (1) and (2) are given in the Appendix A. The two solutions imply two propagation modes, even and odd, that is, symmetric and antisymmetric modes in the SFS waveguide. The symmetric and antisymmetric modes refer to the symmetry or antisymmetry of the pressure and transverse velocity fields $p(y)$ and $v_{y}(y)$ as a function of $y$ within the fluid region. When the air gap between the corrugated plates is large, the SFS waveguide modes split into two single surface acoustic spoof waves, and in the limit $g \longrightarrow \infty$, both Eqs. (1) and (2) become

$$
\beta=k_{0} \sqrt{1+\left(\frac{a}{d}\right)^{2} \tan ^{2}\left(k_{0} h\right)},
$$

which is the dispersion relation of a single surface acoustic spoof wave [24].

To validate the analytical solution given by Eqs. (1) and (2), full-wave FEM simulations are performed using COMSOL Multiphysics 5.3, eigenfrequency simulation for a mode analysis, and frequency domain simulation for the wave propagation analysis. The geometrical parameters are chosen as follows: $d=8, a=5, h=24$, and $g=5 \mathrm{~mm}$, (a)

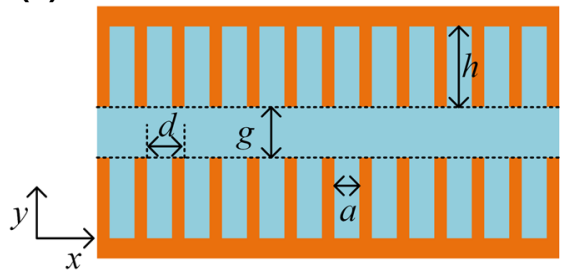

(b)

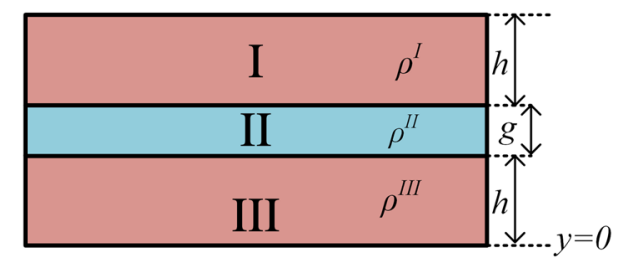

FIG. 1. (a) Structure of the waveguide under analysis, formed by two corrugated rigid plates separated by an air gap of height $g$. (b) Equivalent threelayer structure with effective material parameters. 
(a)

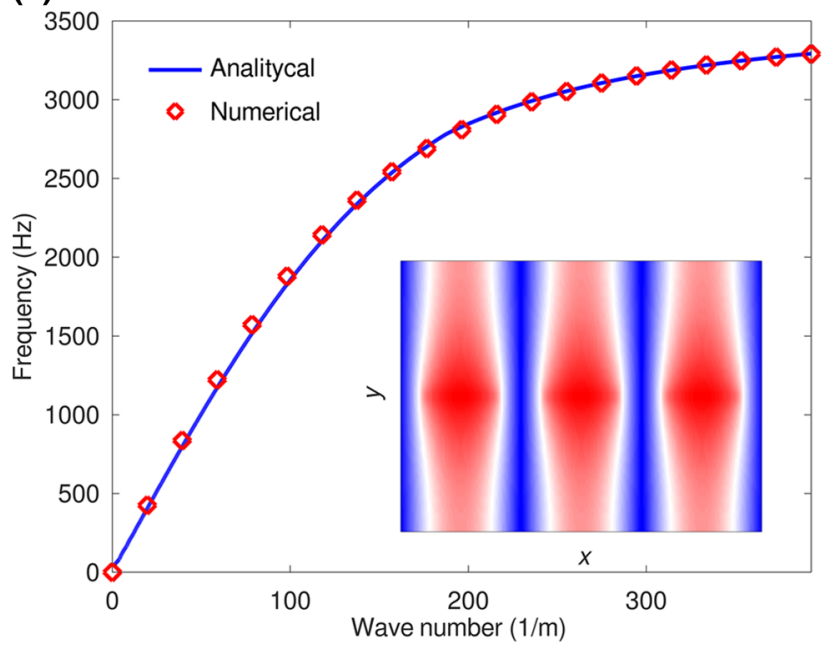

(b)

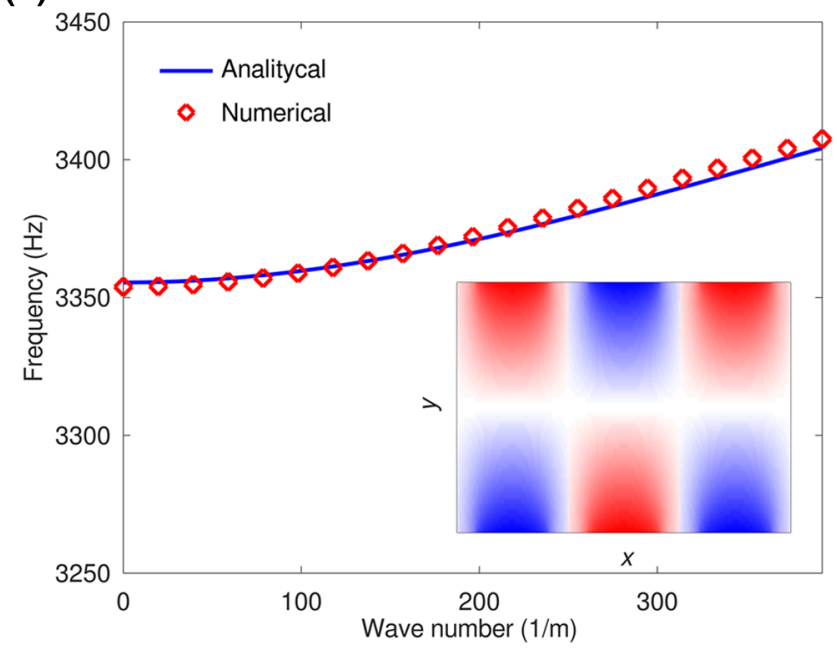

FIG. 2. Analytically and numerically obtained dispersion curve of (a) symmetric and (b) antisymmetric modes. The insets show the spatial pressure distribution inside the waveguide for the corresponding modes when $\beta=1001 / \mathrm{m}$.

where the gap dimension is chosen to ensure that the two acoustic surface modes on the corrugated surfaces are sufficiently coupled. In the numerical simulations, air is used for the fluid medium, which is defined as a linear elastic medium with atmospheric pressure $p_{a}=101.325 \mathrm{kPa}$ and temperature $T=293.15 \mathrm{~K}$. The corrugated surface is modeled as a medium with perfectly rigid walls. Plane wave excitation at the boundary is used as a sound source in the frequency domain and the thermoviscous effects are not included in the FEM simulation since the thermoviscous boundary layer is approximately $100 \mu \mathrm{m}$ at $1 \mathrm{kHz}$ and for the given dimensions has negligible effect.

(a)

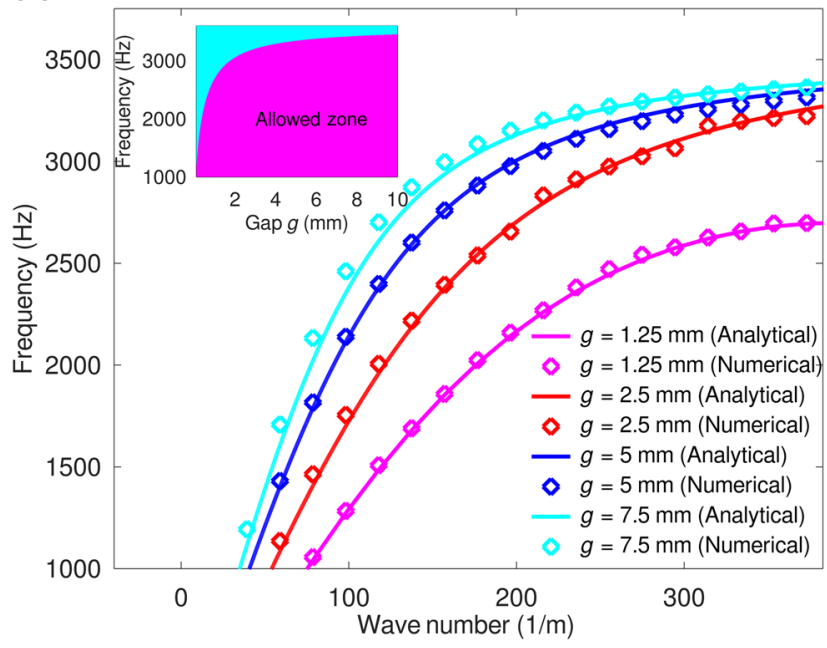

Figures 2(a) and (b) show the analytically and numerically obtained results for symmetric and antisymmetric modes, respectively, for the SFS waveguide. The figure insets show the corresponding spatial distribution of the acoustic pressure within the waveguide for each mode.

An excellent agreement can be observed between analytical and numerical results. The acoustic pressure field distribution inside the waveguide shows the expected characteristics for both modes: in the symmetric case, the field distribution along the $y$ direction is symmetrical, while the antisymmetric mode exhibits antisymmetric features. The symmetric mode is the dominant mode in the SFS waveguide, which does not have a cut-off frequency and its

(b)

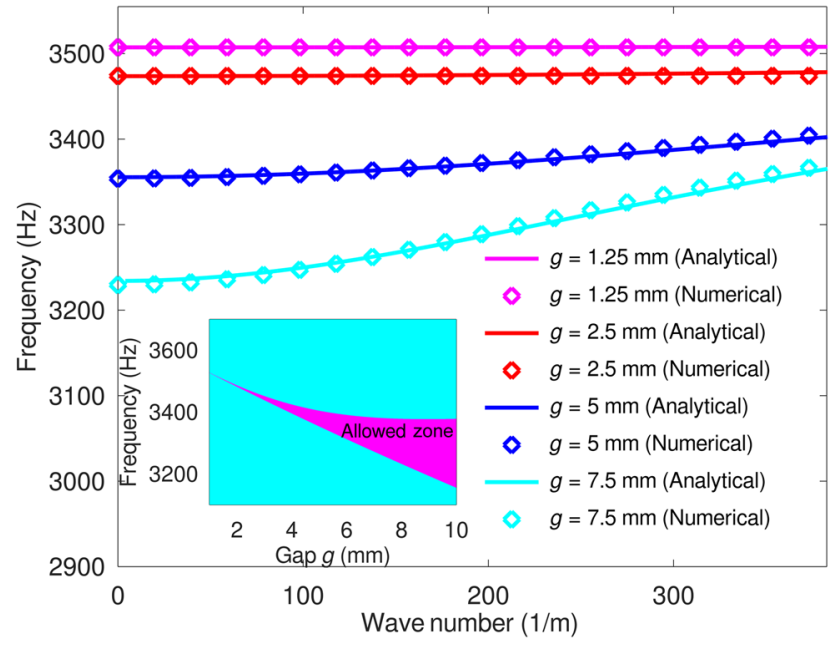

FIG. 3. Analytically and numerically obtained dispersion curve of (a) symmetric and (b) antisymmetric modes for different values of the gap size. The insets show the allowed spectral zones (magenta) and forbidden spectral zones (cyan) for each mode, as a function of the gap size. 
(a)

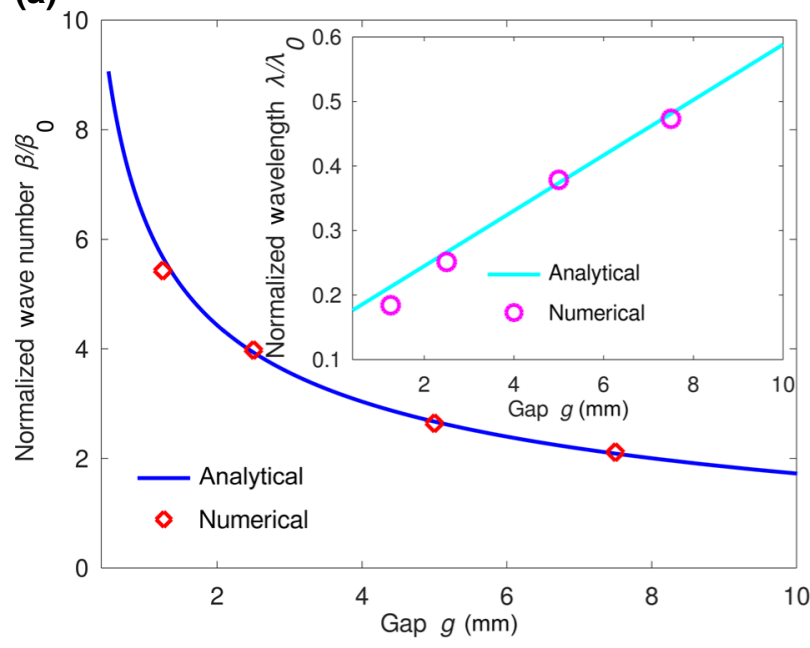

(c)

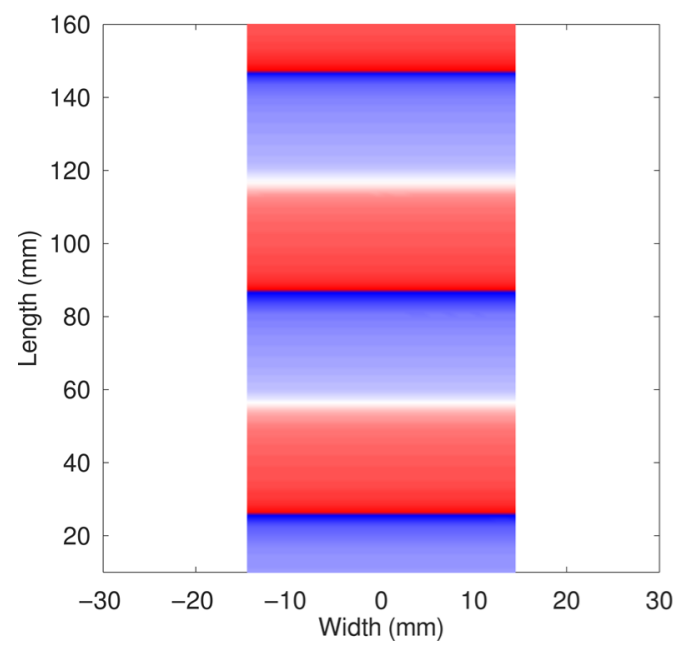

(b)

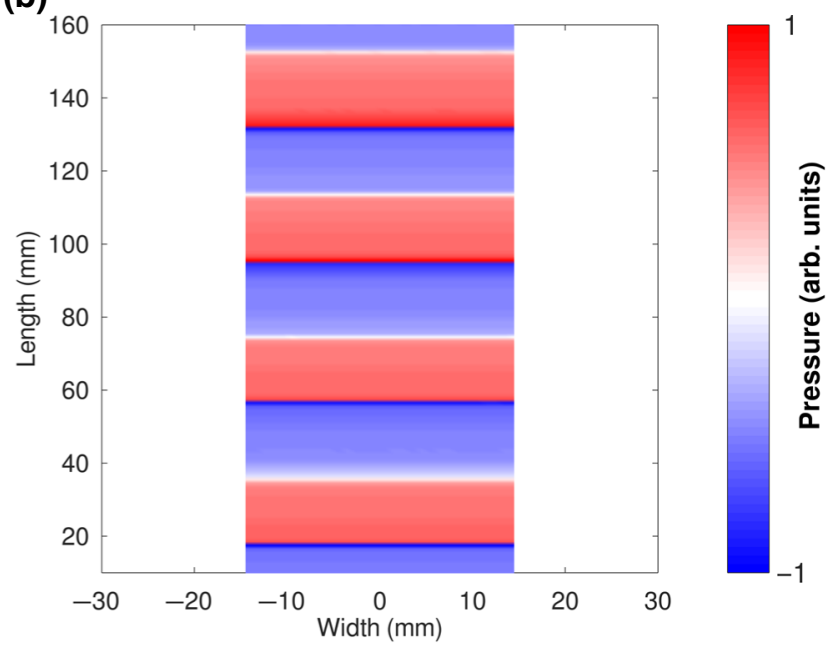

(d)

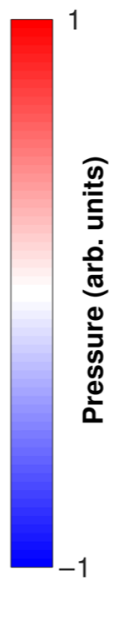

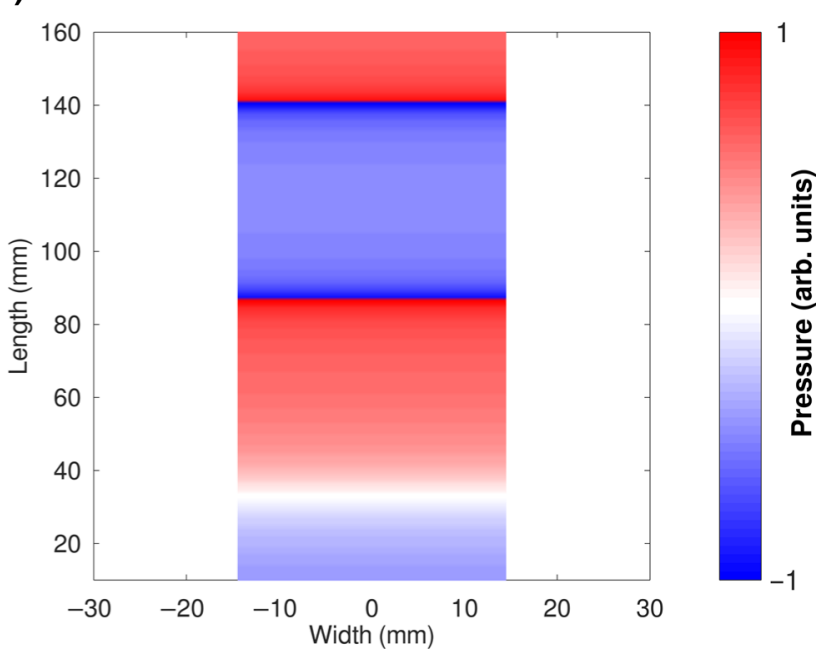

FIG. 4. (a) Normalized wave number as a function of air-gap height between the corrugated surfaces; analytical and simulated wavelengths as a function of the gap heights are shown in the inset. Simulated acoustic pressure distribution along the structure at the operating frequency $f=2000 \mathrm{~Hz}$ for different values of $g$ : (b) $g=1.25 \mathrm{~mm}$, (c) $g=5 \mathrm{~mm}$, and (d) single corrugate surface $(g \longrightarrow \infty$.)

propagation is allowed in the lower part of the frequency spectrum. Namely, the SFS symmetric mode is weakly dispersive at low frequencies, that is, the wave number linearly varies with frequency, however, as frequency increases, strong dispersion occurs and the effective wave number asymptotically approaches infinity. Nevertheless, due to the finite size of the period $d>0$, the propagation constant has a maximum at $\beta=\pi / d$, which corresponds to $3931 / \mathrm{m}$, beyond which the mode hits a stopband. The second mode is antisymmetric, with a cut-off frequency around $3.35 \mathrm{kHz}$. This mode has a very flat dispersion, implying slow sound waves.

A smaller gap induces a smaller group velocity for the symmetric mode, and the maximal frequency that is supported in the SFS waveguide correspondingly decreases. Similarly, the antisymmetric mode becomes even flatter as the gap height decreases, causing a corresponding narrowing of the operating frequency range. To illustrate these effects, we show in Figs. 3(a) and 3(b) how the dispersion properties of the symmetric and antisymmetric modes are controlled by the gap thickness. In addition, the figure insets show the allowed propagation zone for each mode as a function of the gap size. They are calculated from the corresponding dispersion relations under the conditions that the propagation constant is real in the range from 0 to $\pi / d$. Such sensitivity and extreme slow-wave features, especially for the antisymmetric mode, makes the proposed acoustic waveguide a good candidate for acoustic wave modulation and sensing applications. While the antisymmetric mode supports truly extreme dispersion features, in practice, the symmetric mode may be more easily excited and detected. Working with the antisymmetric mode may also be impacted by the presence of localized groove resonances very close in the spectrum. 
(a)

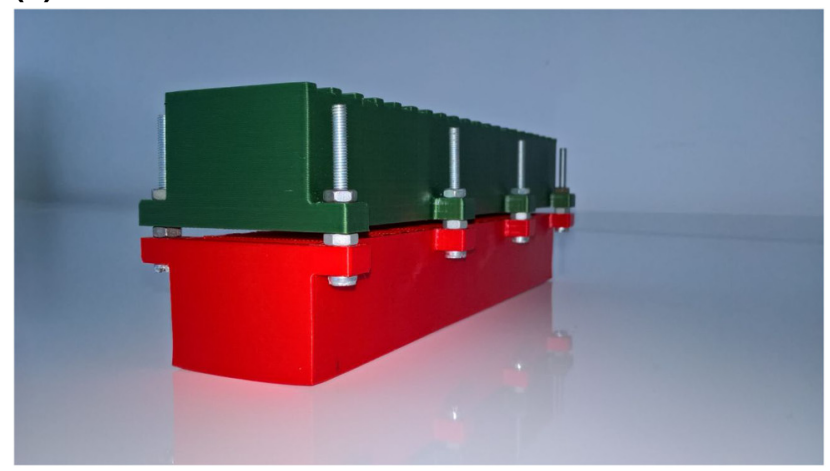

(c)

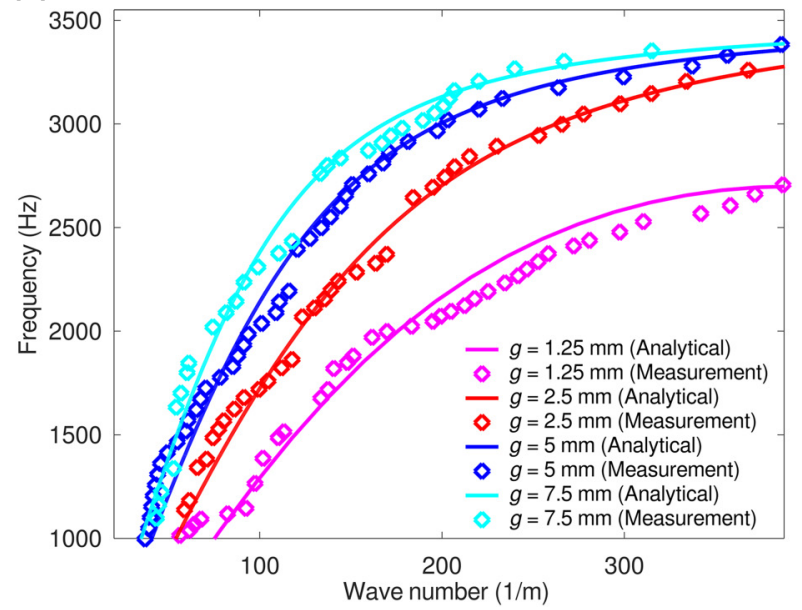

(b)

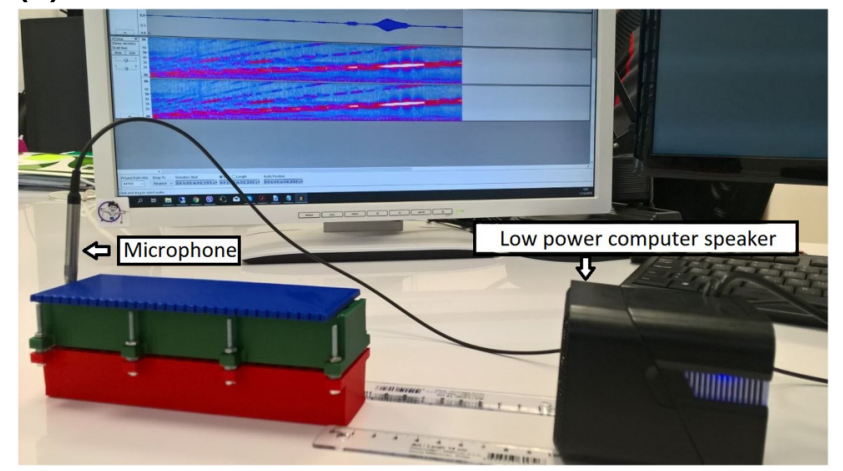

(d)

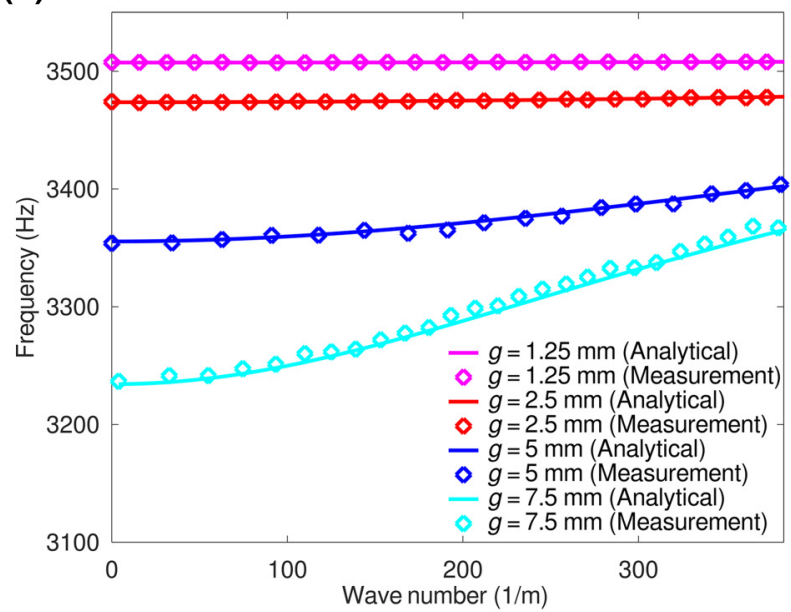

FIG. 5. (a) The assembled prototype of the SFS waveguide. (b) Measurement setup; Measured dispersion curve of the structure with different heights of the air gap between corrugated plates compared with analytical results for (c) symmetric and (d) antisymmetric modes.

Taking this into account, we focus in the following on the excitation of the symmetric mode in the upper region of its dispersion branch, where slow-wave features are found. Figure 4(a) shows the wave number of the symmetric mode normalized to the wave number in air as a function of the gap height $g$ at the frequency $f=2000 \mathrm{~Hz}$. This frequency is chosen since it is located in the frequency range below the existence of the antisymmetric mode, while at the same time, the mode sensitivity is very strong.

It can be noted that the dispersion is sensitive to gap height changes, and this is further demonstrated in Figs. 4(b) -4(d) that show the acoustic pressure field distribution for different values of $g$ and the case of $g \longrightarrow \infty$. It can be concluded that the wavelength significantly decreases as the gap height decreases. In addition, the inset of Fig. 4(a) shows the simulated values of the guided normalized wavelengths versus the height of the air gap. The simulated results follow a linear function given by Eq. (3), which is derived from Eq. (1) using a Tylor series expansion for small $g$. This relation implies that the wavelength in the waveguide can be straightforwardly controlled by the gap height.

$$
\frac{\lambda}{\lambda_{0}} \approx \frac{4 \pi}{\sqrt{3} \lambda_{0}} g+\frac{1}{2 \pi}
$$

The previous analysis shows that the modal dispersion of the SFS waveguide is very sensitive to the gap height, and according to our theoretical analysis, this behavior can also be expected if other geometrical and/or fluid parameters are varied. This indicates a strong potential of the proposed structure for acoustic wave modulation or sensing. For instance, dispersion and the group velocity in the SFS waveguide can be dynamically changed by mechanically adjusting the gap $g$, creating a tunable delay line, while extreme sensing features can be achieved if the sensed analyte is the fluid in the gap. Such possibilities will be the subject of future work.

\section{FABRICATION AND MEASUREMENT}

To verify the analytical and numerical results, an SFS waveguide prototype is fabricated using $3 \mathrm{D}$ printing 
(a)

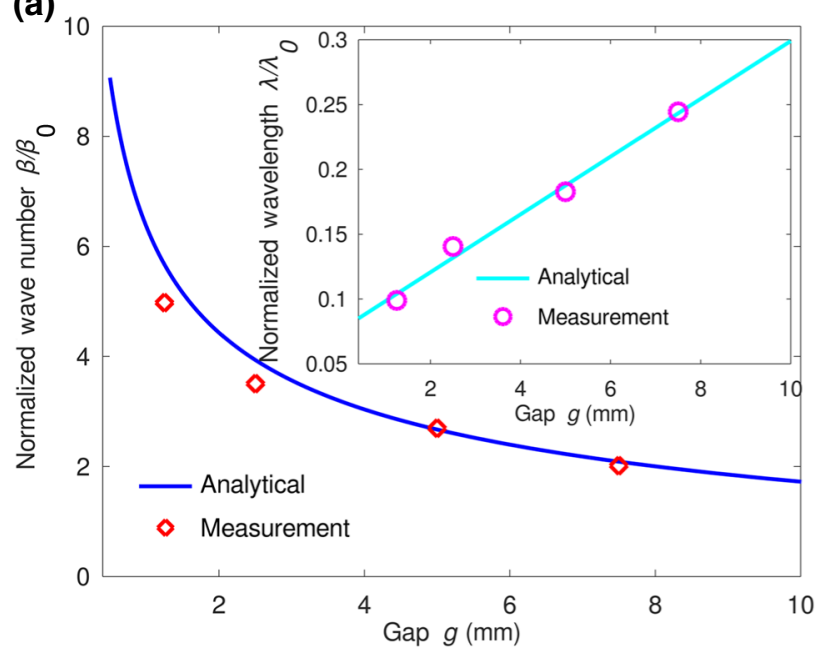

(c)
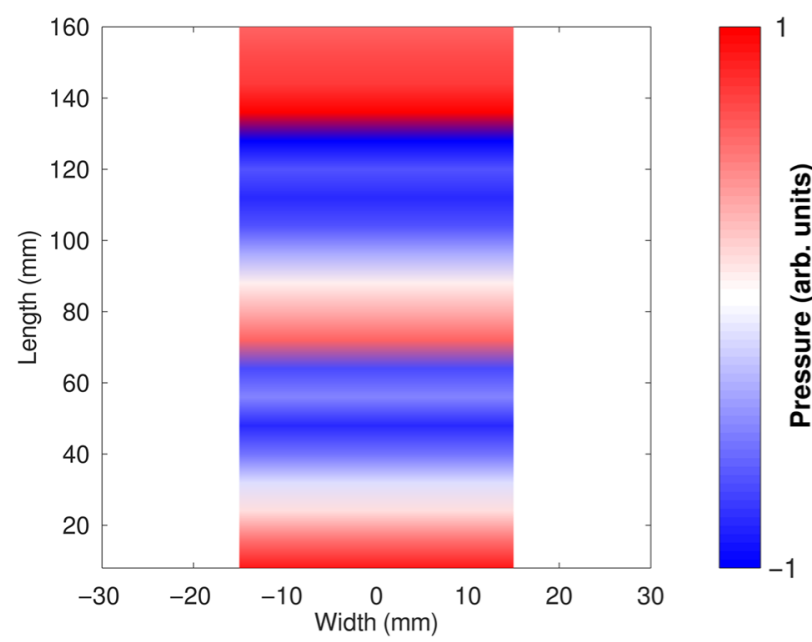

(b)

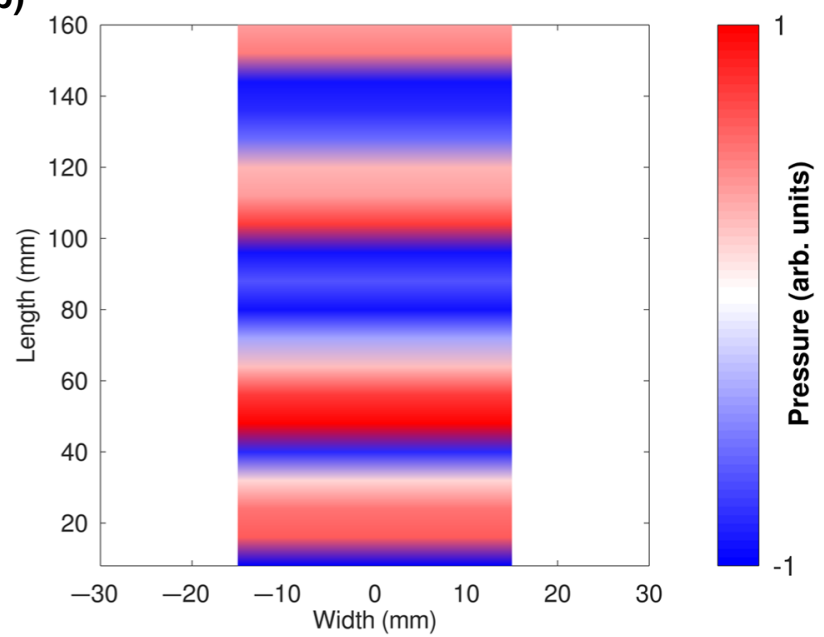

(d)

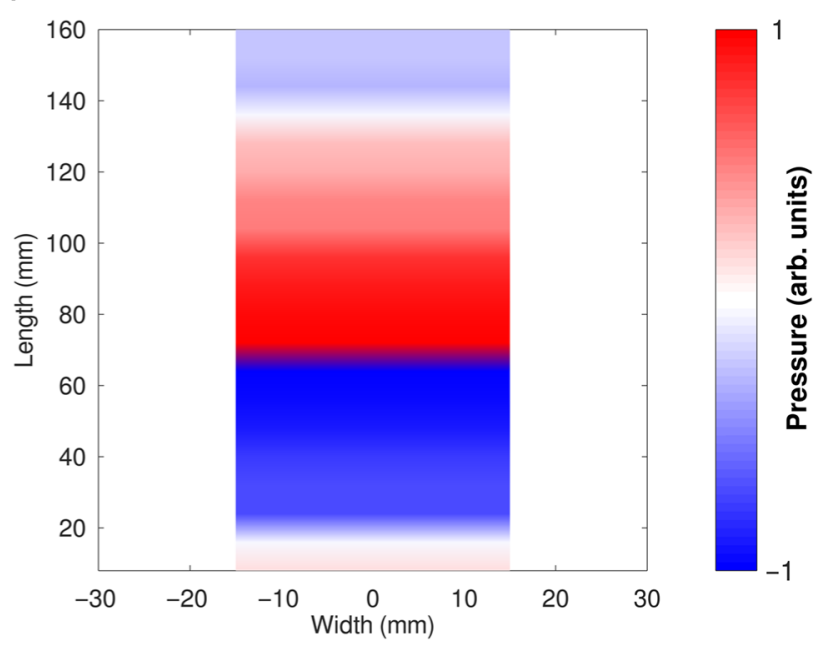

FIG. 6. (a) Analytical and measured wave numbers as a function of the air-gap height between the corrugated surfaces. Analytical and measured wavelengths as a function of the gap height are shown in the inset. Measured acoustic pressure distribution along the structure at the operating frequency $f=2000 \mathrm{~Hz}$ for different values of $g$ : (b) $g=1.25 \mathrm{~mm}$, (c) $g=5 \mathrm{~mm}$, and (d) single corrugate surface $(g \longrightarrow \infty)$

technology-Felix 3.0 3D printer and polylactic acid (PLA) filament material. The PLA acoustic impedance is much larger than the acoustic impedance of air, and due to this large mismatch, the acoustic-solid interaction can be neglected Fig. 5(a). The geometrical parameters of the fabricated structure are $d=8, a=5, h=24$, width $w=30$, and the overall length $L=160 \mathrm{~mm}$. The width and length are chosen so that the fabricated prototype can well correspond to the analyzed 2D structure. Each corrugated part is fabricated separately and then assembled into the final structure using four screws on each side. The air-gap height is controlled by discs with known thickness, which are inserted between the two corrugated parts.

Measurements are performed in a controlled environment in which background pressure and temperature are controlled to make sure that the reflected waves from the room walls are minimized. As a sound source, we use a simple low-power computer speaker, which produces spherical acoustic waves. The source is placed at a $10-\mathrm{cm}$ distance from the structure under test to reduce the distortion between the spherical wave source and the plane wave source used in simulations. The excitation signal is a 30-s-long linear chirp signal with constant amplitude and instantaneous frequency ranging from 0.5 to $3.6 \mathrm{kHz}$. Response of the system is recorded by a G.R.A.S. 46DP $1 / 8$ in. microphone using an external sound card Sound Blaster Omni Surround 5.1 and Audacity software. The sampling frequency is $96 \mathrm{kHz}$, and the obtained signals are postprocessed to recover their amplitudes and phases. The microphone is mounted through small holes in the structure from the top and aligned with the bottom surface of the upper corrugated plates. To obtain the wave numbers, 
the measurement is repeated for different microphone positions with the position difference $\Delta x=8 \mathrm{~mm}$, and the phase difference between the two measurements is used to calculate the wave numbers. The measurement setup and the measured dispersion relation compared to the analytical results are shown in Figs. 5(b)-5(d), respectively.

As discussed in the previous section, the dispersion significantly depends on the gap height, and thus the corresponding measurements are performed to validate the analytical and numerical results. The measurements are repeated with different gap heights between the corrugated surfaces as well as for the case of the spoof plasmon waveguide where one can note a very good agreement between the analytical and experimental results. Additionally, the calculated wave number for the symmetric mode from Fig. 4(a) and the corresponding measured values are compared in Fig. 6(a) and they are in good agreement. Moreover, the inset shows the measured values of the normalized guided wavelengths versus the height of the air gap, confirming our previous theoretical and numerical results.

Finally, we also measure the acoustic pressure field distribution along the SFS waveguide. Acoustic pressure field distributions are measured using the same measurement method described above. The measurement is repeated over the length of the structure with microphone position steps of $8 \mathrm{~mm}$. In this manner, the amplitude and phase of the acoustic pressure are obtained, consequently providing the field distribution. Figures 6(b)-6(d) shows the measured acoustic pressure field distribution for the acoustic surface wave above the single corrugated surface, which corresponds to $g \longrightarrow \infty$, and for the SFS waveguide with the gap heights $g=5$ and $g=1.25 \mathrm{~mm}$. The measured acoustic pressure field distribution corresponds very well to those in Figs. 4(b)-4(d). To conclude, the measurement results confirm the previously shown analytical and numerical results, further demonstrating the potential of the proposed SFS structure for acoustic wave modulation and sensing applications. Moreover, the flat dispersion property of the antisymmetric mode of the proposed structure and its finite cut-off frequency can be exploited as a near-zero medium, which implies a number of possible applications, such as acoustic super coupling and extreme nonreciprocity $[32,33]$. These possibilities will be investigated in the future.

\section{CONCLUSIONS}

We present the acoustic SFS acoustic waveguide, which is an analogue of the MIM plasmonic waveguide. The proposed structure, consisting of two corrugated rigid surfaces separated by a fluid layer, is analyzed in terms of its dispersion and modal properties and sensitivity to the geometrical parameters. The proposed structure is fabricated and measured, and the analytical and numerical results are shown to be in excellent agreement with the measurements. In particular, we focus on the fact that the waveguide dispersion is highly dependent on the gap height, and consequently demonstrate the potential of the proposed structure for acoustic wave modulation and sensing. For instance, dispersion and group velocity in the SFS waveguide can be dynamically changed by mechanically adjusting the gap height, creating a tunable delay line, while a good sensing performance can be achieved if the sensed analyte is the fluid in the gap. Such possibilities will be the subject of future work.

\section{ACKNOWLEDGMENTS}

The work described in this paper has been conducted within the project NOCTURNO that has received funding from the European Union's Horizon 2020 research and innovation programme under Grant No. 777714.

\section{APPENDIX A: DERIVATION OF EQS. (1) AND (2) FROM THE MANUSCRIPT}

The corresponding governing wave equation in the SFS waveguide is

$$
\left[\left(\frac{1}{\rho_{x}}\right) \frac{\partial^{2}}{\partial x^{2}}+\left(\frac{1}{\rho_{y}}\right) \frac{\partial^{2}}{\partial y^{2}}+\frac{\omega^{2}}{\kappa}\right] p=0
$$

where $\omega$ is the angular frequency and $p$ is the acoustic pressure field. The general solution of the wave equation for any layer can be written in the form

$$
p^{j}=\left(A_{+}^{j} e^{y k_{y}^{j}}+A_{-}^{j} e^{-y k_{y}^{j}}\right) e^{-i \beta x},
$$

where $p^{j}$ is the pressure field, $A_{+}^{j}$ and $A_{-}^{j}$ are the amplitudes of the transverse oscillating fields, $k_{y}^{j}$ is the wavevector component along the $y$ direction, and $\beta$ is the propagation constant along the $x$ direction. The $y$ component of the corresponding velocity field for all layers can be calculated from the acoustic pressure as

$$
v_{y}^{j}=\frac{i}{\omega \rho^{j}} \frac{\partial p^{j}}{\partial y}
$$

As stated above, layer I from the top side, $y=b$, and layer III from the bottom side, $y=0$, are assumed to be bounded by perfectly rigid walls. Imposing boundary conditions for the rigid wall, that is, requiring that the normal velocity field on the rigid surface is equal to zero, $v_{y}=0$, the acoustic pressures and the velocity fields in layers I and III can 
be written as

$$
\begin{aligned}
p^{\mathrm{I}} & =2 A_{+}^{\mathrm{I}} \cos \left(y k_{y}^{\mathrm{I}}\right), \\
v_{y}^{\mathrm{I}} & =2 A_{+}^{\mathrm{I}} \frac{i k_{y}^{\mathrm{I}}}{\omega \rho^{\mathrm{I}}} \sin \left(y k_{y}^{\mathrm{I}}\right), \\
p^{\mathrm{III}} & =2 A_{+}^{\mathrm{III}} \cos \left([b-y] k_{y}^{\mathrm{III}}\right), \\
v_{y}^{\mathrm{III}} & =-2 A_{+}^{\mathrm{III}} \frac{i k_{y}^{\mathrm{III}}}{\omega \rho^{\mathrm{III}}} \sin \left([b-y] k_{y}^{\mathrm{III}}\right),
\end{aligned}
$$

where $\omega$ is the angular frequency, $b$ is the overall height of the three-layer structure, and $\rho^{\mathrm{I}}$ and $\rho^{\mathrm{III}}$ are the finite effective material mass densities for layers I and III, respectively. The parameters $k_{y}^{\mathrm{I}}=\omega \sqrt{\rho^{\mathrm{I}} / \kappa}$ and $k_{y}^{\mathrm{III}}=\omega \sqrt{\rho^{\mathrm{III}} / \kappa}$ are transverse wavevector components in layers I and III, respectively. In the fluid layer, that is, the air gap, the acoustic pressure field and the corresponding velocity field can be derived from Eq. (A1) as

$$
\begin{aligned}
p^{\mathrm{II}} & =\Upsilon \cosh \left(\left[y-h-\frac{g}{2}\right] k_{y}^{\mathrm{II}}+\psi\right), \\
v_{y}^{\mathrm{II}} & =\frac{i \Upsilon k_{y}^{\mathrm{II}}}{\omega \rho^{\mathrm{II}}} \Upsilon \sinh \left(\left[y-h-\frac{g}{2}\right] k_{y}^{\mathrm{II}}+\psi\right) .
\end{aligned}
$$

The parameters $\Upsilon$ and $\psi$ can be calculated from the amplitudes of the transversely oscillating fields in layer II as $\Upsilon=\sqrt{\left(A_{+}^{\mathrm{II}}+A_{-}^{\mathrm{II}}\right)^{2}-\left(A_{+}^{\mathrm{II}}-A_{-}^{\mathrm{II}}\right)^{2}}$ and $\psi=\tanh ^{-1}\left[\left(A_{+}^{\mathrm{II}}-\right.\right.$ $\left.\left.A_{-}^{\mathrm{II}}\right) /\left(A_{+}^{\mathrm{II}}+A_{-}^{\mathrm{II}}\right)\right]$, where $h$ and $g$ are the geometrical parameters shown in Fig. 1(a), $\rho^{\mathrm{III}}$ is the density of the fluid, and $k_{y}^{\mathrm{II}}$ is the transversal wavevector component along $y$. The transverse wave vector is expressed as $k_{y}^{\mathrm{II}}=$ $\sqrt{\beta^{2}-k_{0}^{2}}$, where $k_{0}=\omega \sqrt{\rho_{\text {air }} / \kappa_{\text {air }}}$ is the wave number in the fluid, and $\beta$ is the propagation constant in the SFS waveguide. Applying the boundary conditions, where the pressure field $p$ and normal velocity component $v_{y}$ are continuous at the interfaces between different layers, $y=h$ and $y=h+g$, we obtain the dispersion equations for the guided modes in the SFS acoustic waveguide

$$
\begin{aligned}
& \frac{k_{y}^{\mathrm{I}}}{\rho^{\mathrm{I}}} \tan \left(h k_{y}^{\mathrm{I}}\right)=\frac{k_{y}^{\mathrm{II}}}{\rho^{\mathrm{II}}} \tanh \left(\frac{g}{2} k_{y}^{\mathrm{II}}-\psi\right), \\
& \frac{k_{y}^{\mathrm{III}}}{\rho^{\mathrm{III}}} \tan \left(h k_{y}^{\mathrm{III}}\right)=\frac{k_{y}^{\mathrm{II}}}{\rho^{\mathrm{II}}} \tanh \left(\frac{g}{2} k_{y}^{\mathrm{II}}+\psi\right) .
\end{aligned}
$$

When the upper and lower corrugated surfaces have the same geometrical parameters, the effective material parameters are also the same. Therefore, the transverse wavevector components are equal $k_{y}^{\mathrm{I}}=k_{y}^{\mathrm{III}}$, which implies two possible solutions: $\psi=0$ and $\psi=i \pi / 2$. Using the identity $\tanh (x \pm i \pi / 2)=\operatorname{coth}(x)$ and Eqs. (A6a) and (A6b), the dispersion relations become Eqs. (1) and (2) in the main manuscript.

[1] S. Maier, Plasmonics: Fundamentals and Applications (Springer Science \& Business Media, Springer US, New York, 2007).

[2] Y. Li, Plasmonic Optics: Theory and Applications (SPIE Press, Bellingham, Washington, 2017).

[3] L. Xiangang and Y. Lianshan, Surface plasmon polaritons and its applications, IEEE Photonics J. 4, 590 (2012).

[4] J. Wang, W. Lin, E. Cao, X. Xu, W. Liang, and X. Zhang, Surface plasmon resonance sensors on Raman and fuorescence spectroscopy, Sensors 17, 2719 (2017).

[5] P. Singh, SPR Biosensors: Historical perspectives and current challenges, Sens. Actuators B 229, 110 (2016).

[6] J. B. Pendry, L. Martín-Moreno, and F. J. Garcia-Vidal, Mimicking surface plasmons with structured surfaces, Science 305, 847 (2004).

[7] A. P. Hibbins, B. R. Evans, and J. R. Sambles, Experimental verification of designer surface plasmons, Science 308, 670 (2005).

[8] E. N. Economou, Surface plasmons in thin films, Phys. Rev. 182, 539 (1969).

[9] V. R. Almeida, Q. Xu, C. A. Barrios, and M. Lipson, Guiding and confining light in void nanostructure, Opt. Lett. 29, 1209 (2004).

[10] J. A. Dionne, L. A. Sweatlock, H. A. Atwater, and A. Polman, Plasmon slot waveguides: Towards chip-scale propagation with subwavelength-scale localization, Phys. Rev. B 73, 035407 (2006).

[11] M. A. Kats, D. Woolf, R. Blanchard, N. Yu, and F. Capasso, Spoof plasmon analogue of metal-insulator-metal waveguides, Opt. Express 19, 14860 (2011).

[12] D. Woolf, M. A. Kats, and F. Capasso, Spoof surface plasmon waveguide forces, Opt. Letters 39, 517 (2014).

[13] L. Kelders, J. F. Allard, and W. Lauriks, Ultrasonic surface waves above rectangular-groove gratings, J. Acoust. Soc. Am. 103, 2730 (1998).

[14] L. Kelders, W. Lauriks, and J. F. Allard, Surface waves above thin porous layers saturated by air at ultrasonic frequencies, J. Acoust. Soc. Am. 104, 882 (1998).

[15] J. Christensen, A. I. Fernandez-Dominguez, F. de LeonPerez, L. Martin-Moreno, and F. J. Garcia-Vidal, Collimation of sound assisted by acoustic surface waves, Nat. Phys. 3, 851 (2007).

[16] Z. He, H. Jia, C. Qiu, Y. Ye, R. Hao, M. Ke, and Z. Liu, Nonleaky surface acoustic wave s on a textured rigid surface, Phys. Rev. B 83, 132101 (2011).

[17] J. Christensen, L. Martín-Moreno, and F. J. García-Vidal, Enhanced acoustical transmission and beaming effect through a single aperture, Phys. Rev. B 81, 174104 (2010).

[18] J. Christensen, P. A. Huidobro, L. Martín-Moreno, and F. J. García-Vidal, Confining and slowing airborne sound with a corrugated metawire, Appl. Phys. Lett. 93, 083502 (2008).

[19] Z. Yu, M.-H. Lu, L. Feng, X. Ni, Y.-F. Chen, Y.-Y. Zhu, S.-N. Zhu, and N.-B. Ming, Acoustic Surface Evanescent Wave and its Dominant Contribution to Extraordinary Acoustic Transmission and Collimation of Sound, Phys. Rev. Lett. 104, 164301 (2010). 
[20] J. Zhu, Y. Chen, X. Zhu, F. J. Garcia-Vidal, X. Yin, W. Zhang, and $X$. Zhang, Acoustic rainbow trapping, Sci. Rep. 3, 1728 (2013).

[21] H. Jia, M. Lu, X. Ni, M. Bao, and X. Li, Spatial separation of spoof surface acoustic waves on the graded groove grating, J. Appl. Phys. 116, 124504 (2014).

[22] Y. Chen, H. Liu, M. Reilly, H. Bae, and M. Yu, Enhanced acoustic sensing through wave compression and pressure amplification in anisotropic metamaterials, Nat. Commun. 5, 5247 (2014).

[23] Y. Ye, M. Ke, Y. Li, T. Wang, and Z. Liu, Focusing of spoof surface-acoustic-waves by a gradient-index structure, J. Appl. Phys. 114, 154504 (2013).

[24] B. I. Popa and S. A. Cummer, Design and characterization of broadband acoustic composite metamaterials, Phys. Rev. B 80, 174303 (2009).

[25] A. Climente, D. Torrent, and J. Sánchez-Dehesa, Sound focusing by gradient index sonic lenses, Appl. Phys. Lett. 97, 104103 (2010).

[26] S. Xie, S. Ouyang, Z. He, X. Wang, K. Deng, and H. Zhao, Bending and splitting of spoof surface acoustic waves through structured rigid surface, Result Phys. 8, 52 (2018).
[27] C. Li, M. Ke, S. Zhang, S. Peng, C. Qiu and Z. Liu, Guiding spoof surface acoustic waves on a monolayer array of rigid cylinders in water, J. Phys. D: Appl. Phys. 49, 125304 (2016).

[28] L. T. Wu, G. Y. Song, W. K. Cao, Q. Cheng, T. J. Cui, and Y. Jing, Generation of multiband spoof surface acoustic waves via high-order modes, Phys. Rev. B 97, 214305 (2018).

[29] N. Cselyuszka, M. Sečujski, N. Engheta, and V. CrnojevićBengin, Temperature-controlled acoustic surface waves, New J. Phys. 18, 103006 (2016).

[30] L. Rayleigh, On waves propagated along the plane surface of an elastic solid, Proc. Lond. Math. Soc. 17 (1885).

[31] F. J. Garcia-Vidal, L. Martýn-Moreno, and J. B. Pendry, Surfaces with holes in them: New plasmonic metamaterials, J. Opt. A: Pure Appl. Opt. 7, S97 (2005).

[32] H. Esfahlani, M. S. Byrne, M. McDermott, and A. Alù, Acoustic supercoupling in a zero-compressibility waveguide, Research 2016, 2457870 (2019).

[33] L. Quan, D. L. Sounas, and A. Alù, Non-Reciprocal Willis Coupling in Zero-Index Moving Media, Phys. Rev. Lett. 123, 064301 (2019). 